Also available at http://amc.imfm.si

ISSN 1855-3966 (printed edn.), ISSN 1855-3974 (electronic edn.)

ARS MATHEMATICA CONTEMPORANEA 5 (2012) 295-306

\title{
Side lengths of pseudoconvex fullerene patches
}

\author{
Christina Graves , Jennifer McLoud-Mann \\ The University of Texas at Tyler, Department of Mathematics, Tyler, TX 75799, USA
}

Received 20 September 2011, accepted 11 November 2011, published online 29 March 2012

\begin{abstract}
In this paper we consider fullerene patches with nice boundaries containing between one and five pentagonal faces. We find necessary conditions for the side lengths of such patches, and then prove these conditions are sufficient by constructing such patches.
\end{abstract}

Keywords: Fullerenes, fullerene patches, boundary codes, pseudoconvex patches.

Math. Subj. Class.: 05C10, 05C75, 92 E10

\section{Preliminaries}

Mathematically speaking, a fullerene is a trivalent plane graph having only hexagonal and pentagonal faces. As a consequence of Euler's formula, such a graph has exactly 12 pentagons. A fullerene patch is formed by taking a simple closed curve on a fullerene and deleting all vertices and edges on one side of the curve. The simple curve used to create the patch will be called the boundary. Equivalently, we can think of a fullerene patch as being a plane graph having only hexagonal and at most 12 pentagonal faces in addition to one external face with all internal vertices having valence 3 and all vertices on the the external face having valence 2 or 3 . Such patches have been studied in [2], [3], and [7]. These patches are also closely related to nanocone tips (see [3] and [9]).

The boundary code of a fullerene patch is described by a sequence of 2's and 3's corresponding to the valence of the vertices on the boundary in cyclic order. Boundary codes with the same cyclic permutation (same sequence starting at a different vertex) are equivalent. In addition, boundary codes represented by a cyclic permutation and its inverse (changing clockwise to counterclockwise orientation or vice versa) are equivalent. Boundary codes have been studied in [6], [8], and [5] and there have been many algorithms that decide whether a given boundary code is permissible (see [4] and [1]).

In this paper, we limit our study to fullerene patches with nice boundaries. Adopting the terminology from [3], we summarize a few important definitions and include some of our own. A pseudoconvex patch is a fullerene patch where the boundary code does not

E-mail addresses: cgraves@uttyler.edu (Christina Graves),jmcloud@uttyler.edu (Jennifer McLoud-Mann) 
contain the subsequence $(3,3)$. In a pseudoconvex patch, a break edge is an edge on the boundary that has two vertices of degree 2 . The section of the boundary between two break edges is a side of the patch and the length of a side is the number of vertices of degree 3 between the two break edges. A face is a $\left(\ell_{i}, \ell_{j}\right)$-corner if it is the face on both a side of length $\ell_{i}$ and a side of length $\ell_{j}$. In this paper, we are only studying pseudoconvex patches, so we use the term patches if the meaning is clear. Due to Euler's formula, the number of sides and the number of pentagons in a pseudoconvex patch are closely related. [2]

Lemma 1.1. In a pseudoconvex patch, the number of sides $s$ and the number of pentagons $p$ is related by

$$
s=6-p
$$

Because the boundary codes can be quite long with pseudoconvex patches, we use a new method to describe the boundary.

Definition 1.2. The side parameters of a pseudoconvex patch with side lengths $\ell_{1}, \ldots, \ell_{s}$ in cyclic order are given by $\left[\ell_{1}, \ldots, \ell_{s}\right]$.

The side parameters of a psuedoconvex patch come directly from the boundary code by counting the number of 3's between two pairs of consecutive 2's where three 2's in a row correspond to a side of length 0 , and four 2 's in a row correspond to two sides of length 0 . For example, a patch with the boundary code

$$
222(323) 222(3) 22(323)
$$

has side parameters $[0,2,0,1,2]$. To go to the boundary code from side parameters, we follow a similar process. If a patch has $s$ sides and side parameters $\left[\ell_{1}, \ldots, \ell_{s}\right]$, the boundary code is given by

$$
2(23)^{\ell_{1}}(2)(23)^{\ell_{2}} \cdots(2)(23)^{\ell_{s}} .
$$

It was shown in [7] that the side parameters of a patch with only hexagons satisfy certain conditions. It is not hard to follow the proof backward to show that the converse is also true. The result is summarized below.

Lemma 1.3. The sequence of integers $\left[\ell_{1}, \ell_{2}, \ell_{3}, \ell_{4}, \ell_{5}, \ell_{6}\right]$ are the side parameters of a pseudoconvex patch consisting solely of hexagonal faces if and only the the following three equalities are satisfied:

$$
\begin{aligned}
& \text { (1.3.1) } \ell_{1}+\ell_{2}=\ell_{4}+\ell_{5} \\
& \text { (1.3.2) } \ell_{2}+\ell_{3}=\ell_{5}+\ell_{6} \\
& \text { (1.3.3) } \ell_{3}+\ell_{4}=\ell_{6}+\ell_{1}
\end{aligned}
$$

Equivalently, this lemma says that the sequence $2(23)^{\ell_{1}} \cdots 2(23)^{\ell_{6}}$ is the boundary code for a patch if and only if the three equalities above hold.

The goal of the rest of this paper is to continue in the manner of Lemma 1.3 and find necessary and sufficient conditions on the side parameters of any pseudoconvex patch. Note that we only need focus on patches containing zero to five pentagons since a patch containing seven to twelve pentagons is a complement of an aforementioned patch. Many of these proofs involve exploring the effect of adding or deleting rows of hexagons from a given patch. 


\section{Necessary conditions on side parameters}

To find conditions on patches with one or more pentagons, we will use inductive arguments that involve making a patch smaller. We accomplish this by deleting all faces on one side of a patch which will be called stripping off the side. It is clear that stripping off a side results in another pseudoconvex patch [7]. Also, adding a row of hexagons along a side results in another pseudoconvex patch. The following lemma tells us how these processes affect the side parameters of a patch under certain conditions.

Lemma 2.1. Let $\Pi$ be a pseudoconvex patch with side parameters $\left[\ell_{1}, \ldots, \ell_{s}\right]$.

1. If the $\ell_{1}$ side contains only hexagons, $\ell_{2}$ and $\ell_{s}$ are positive, and $s \geq 3$, then stripping off the $\ell_{1}$ side results in a new pseudoconvex patch with parameters

$$
\left[\ell_{1}+1, \ell_{2}-1, \ell_{3}, \ldots, \ell_{s-1}, \ell_{s}-1\right]
$$

2. If $\ell_{1}>0$ and $s \geq 3$, then adding a row of hexagons to the $\ell_{1}$ side results in a new pseudoconvex patch with side parameters

$$
\left[\ell_{1}-1, \ell_{2}+1, \ell_{3}, \ldots, \ell_{s-1}, \ell_{s}+1\right]
$$

There are many extensions of this lemma including what happens if adjacent sides have length 0 , or what happens if the side to strip has a pentagon on it. All arguments are similar, though, and the list would be too extensive to include here. We will be using many of these arguments throughout the rest of the paper.

Because many of the arguments throughout this paper are inductive, a special type of pseudoconvex patch is important to understand. A linear patch is a patch with boundary code

$$
(23)^{\ell_{1}} 2^{r_{1}}(23)^{\ell_{1}} 2^{r_{2}} \text {. }
$$

In other words, a linear patch is a straight chain of adjacent hexagons capped off at each end with either a pentagon or hexagon. Thus, the possible side parameters of a linear patch are $\left[\ell_{1}, 0,0, \ell_{1}, 0,0\right]$ (if both ends are hexagons), $\left[\ell_{1}, 0,0, \ell_{1}, 0\right]$ (if one end is a hexagon and one is a pentagon), and $\left[\ell_{1}, 0, \ell_{1}, 0\right]$ (if both ends are pentagons). In fact, any time a pentagon in a patch has four edges on the boundary, the patch must be linear. Also, any patch whose side parameters contain two consecutive zeros must be linear.

The necessary conditions on the side parameters of a pseudoconvex patch containing exactly one pentagon are stated in the following lemma.

Lemma 2.2. Let $\Pi$ be a pseudoconvex patch with side parameters $\left[\ell_{1}, \ell_{2}, \ell_{3}, \ell_{4}, \ell_{5}\right]$. Then the following inequalities hold:

(2.2.1) $\ell_{1}+\ell_{2} \geq \ell_{4}$

(2.2.2) $\ell_{2}+\ell_{3} \geq \ell_{5}$

(2.2.3) $\ell_{3}+\ell_{4} \geq \ell_{1}$

(2.2.4) $\ell_{4}+\ell_{5} \geq \ell_{2}$

(2.2.5) $\ell_{5}+\ell_{1} \geq \ell_{3}$ 
Proof. Let $\Pi$ be as given. Let $d$ be the minimum distance from the pentagon to a boundary face of $\Pi$ and without loss of generality assume that the pentagon is closest to the $\ell_{1}$ side. We will induct on $d$.

For the base case where $d=0$, the pentagon must be on a boundary face of the patch. If the pentagon is the only face in the patch, then the parameters are $[0,0,0,0,0]$ which clearly satisfy all the inequalities. If the pentagon has four edges on the boundary, then the patch must be linear with parameters $[0, a, 0,0, a]$ and these parameters also satisfy all required inequalities. Notice that any sequence of side parameters that contains two consecutive zeros falls into these categories. Thus our work on the base case begins in earnest when when $\ell_{1}>0$ and there are no two consecutive 0 's.

Let $t$ be the distance from the pentagon to the $\left(\ell_{1}, \ell_{5}\right)$-corner. If neither $\ell_{2}$ nor $\ell_{5}$ are zero, then we strip off the $\ell_{1}$ side to create a new patch containing only hexagons. This patch has side parameters $\left[t, \ell_{1}-t, \ell_{2}-1, \ell_{3}, \ell_{4}, \ell_{5}-1\right]$ (see Figure 1). Using the result from Lemma 1.3, we have the following three equalities:

$$
\begin{aligned}
& \ell_{1}=\ell_{3}+\ell_{4} \\
& \ell_{1}+\ell_{2}=\ell_{4}+\ell_{5}+t \\
& \ell_{2}+\ell_{3}=\ell_{5}+t .
\end{aligned}
$$

Using these equalities, we see that (2.2.1) is a consequence of the middle equality, (2.2.2) is derived from the bottom equality and (2.2.3) comes from top equality. Since $t \leq \ell_{1}$, inequality (2.2.4) comes from the middle equation. Finally, (2.2.5) is obtained by noticing that $\ell_{1}+\ell_{5}=\ell_{3}+\ell_{4}+\ell_{5}$ (from the top equation) which is clearly greater than or equal to $\ell_{3}$.

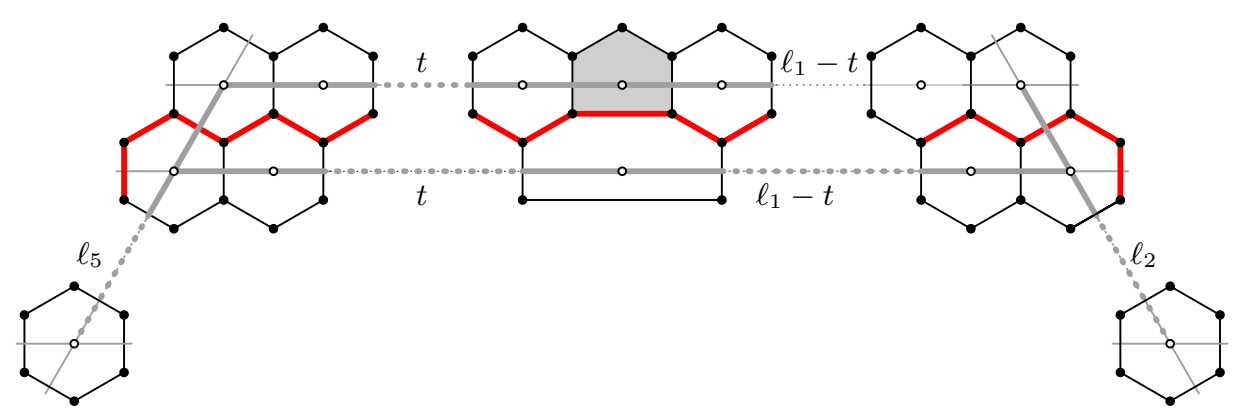

Figure 1: Stripping off a side with a pentagon distance $t$ from the corner and adjacent sides having nonzero length. Red lines indicate the new boundary.

If $\ell_{2}=0$ and $\ell_{5}$ is nonzero, we similarly strip off the $\ell_{1}$ side to create a new patch containing only hexagons. This time, though, the patch has side parameters $\left[t, \ell_{1}-t-\right.$ $\left.1,0, \ell_{3}-1, \ell_{4}, \ell_{5}-1\right]$. We again use the equalities from Lemma 1.3 to get the same three equations as the previous case. Finally, we consider the case where $\ell_{2}=\ell_{5}=0$. If the 
pentagon is on the $\left(\ell_{2}, \ell_{1}\right)$-corner or $\left(\ell_{5}, \ell_{1}\right)$-corner, we refer to the linear case described in the first paragraph of the proof. Otherwise, we again strip off the $\ell_{1}$ side of the patch to create a new patch with only hexagons and side parameters $\left[t-1, \ell_{1}-t-1,0, \ell_{3}-1, \ell_{4}-\right.$ $1,0]$. The three equalities that must be satisfied by this patch are the same as above thus proving the base case.

Now assume that $d$ (the distance from the pentagon to the $\ell_{1}$ side) is greater than 0 . Since the pentagon is closest to the $\ell_{1}$ side, it is clear that $\ell_{1}$ is nonzero. Because the $\ell_{1}$ side is only hexagons, stripping the $\ell_{1}$ side off the patch results in a new patch containing exactly one pentagon. The side parameters of the new patch are:

$$
\begin{array}{r}
{\left[\ell_{1}+1, \ell_{2}-1, \ell_{3}, \ell_{4}, \ell_{5}-1\right] \text { if } \ell_{2} \neq 0, \ell_{5} \neq 0} \\
{\left[\ell_{1}, 0, \ell_{3}-1, \ell_{4}, \ell_{5}-1\right] \text { if } \ell_{2}=0, \ell_{5} \neq 0} \\
{\left[\ell_{1}, \ell_{2}-1, \ell_{3}, \ell_{4}-1,0\right] \text { if } \ell_{2} \neq 0, \ell_{5}=0} \\
{\left[\ell_{1}-1,0, \ell_{3}-1, \ell_{4}-1,0\right] \text { if } \ell_{2}=\ell_{5}=0 .}
\end{array}
$$

In all of these new patches, the distance from the pentagon to the $\ell_{1}$ side is $d-1$, so by induction we know that the inequalities of the lemma are satisfied. It is not hard to list these inequalities and verify that the original patch must have satisfied the conditions as well.

We now list necessary conditions on the side parameters of a pseudoconvex patch containing two pentagons.

Lemma 2.3. Let $\Pi$ be a pseudoconvex patch with side parameters $\left[\ell_{1}, \ell_{2}, \ell_{3}, \ell_{4}\right]$. Then there are no consecutive zeros and the following inequalities must hold.

(2.3.1) $2 \ell_{1}+\ell_{2}+\ell_{4}>\ell_{3}$

(2.3.2) $2 \ell_{2}+\ell_{3}+\ell_{1}>\ell_{4}$

(2.3.3) $2 \ell_{3}+\ell_{4}+\ell_{2}>\ell_{1}$

(2.3.4) $2 \ell_{4}+\ell_{1}+\ell_{3}>\ell_{2}$

Proof. First, notice that two consecutive zeros implies we have a linear patch with a hexagon on one end which is impossible. Let $\Pi$ be a pseudoconvex patch with side paramters $\left[\ell_{1}, \ell_{2}, \ell_{3}, \ell_{4}\right]$ and define the length of $\Pi$ to be $\ell=\ell_{1}+\ell_{2}+\ell_{3}+\ell_{4}$. The remainder of this proof will go by induction on $\ell$. The smallest patch containing two pentagons is the patch with two pentagons touching and no hexagons. Such a patch has side parameters $[1,0,1,0]$ which is length 2 and satisfies the inequalities. The only other sequence that has length 2 is $[2,0,0,0]$ which has two consecutive zeros and is therefore not permissible. Thus the base case is proven.

We now assume that $\ell$ is bigger than 2 . Since $\Pi$ has four sides and two pentagons, either the pentagons are on opposite corners of the patch or there is some side with no pentagons. If the two pentagons on opposite corners, then we can remove the two pentagons resulting in a hexagonal patch with side parameters $\left[1, \ell_{1}-1, \ell_{2}-1,1, \ell_{3}-1, \ell_{4}-1\right]$. This patch must satisfy the equalities given for hexagonal convex patches in Lemma 1.3 , and we see that $\ell_{1}=\ell_{3}$ and $\ell_{2}=\ell_{4}$. Thus

$$
2 \ell_{1}+\ell_{2}+\ell_{4}=2 \ell_{3}+2 \ell_{2}>\ell_{3}
$$


since $\ell_{2}$ and $\ell_{3}$ cannot both be zero. The other inequalities follow similarly.

If the two pentagons are not on opposite corners, there is some side that consists solely of hexagons. Without loss of generality, we will assume this side is the $\ell_{1}$ side. Stripping this side from the patch yields a new patch with parameters

$$
\begin{array}{r}
{\left[\ell_{1}+1, \ell_{2}-1, \ell_{3}, \ell_{4}-1\right] \text { if } \ell_{2} \neq 0, \ell_{4} \neq 0 ;} \\
{\left[\ell_{1}, 0, \ell_{3}-1, \ell_{4}-1\right] \text { if } \ell_{2}=0, \ell_{4} \neq 0 ;} \\
{\left[\ell_{1}, \ell_{2}-1, \ell_{3}-1,0\right] \text { if } \ell_{2} \neq 0, \ell_{4}=0 ;} \\
{\left[\ell_{1}-1,0, \ell_{3}-2,0\right] \text { if } \ell_{2}=\ell_{4}=0 .}
\end{array}
$$

Notice the new total length of all of these patches is less than $\ell$. In the first case, the new length is $\ell-1$ and we use induction to see that the following inequalities hold:

$$
\begin{aligned}
2 \ell_{1}+\ell_{2}+\ell_{4} & >\ell_{3} ; \\
2 \ell_{2}+\ell_{3}+\ell_{1} & >\ell_{4} ; \\
2 \ell_{3}+\ell_{4}+\ell_{2}-3 & >\ell_{1} ; \\
2 \ell_{4}+\ell_{1}+\ell_{3} & >\ell_{2} .
\end{aligned}
$$

Thus the original patch $\Pi$ must have satisfied the inequalities stated in the lemma. The other cases can be done similarly.

Lemma 2.4. Let $\Pi$ be a pseudoconvex patch with side parameters $\left[\ell_{1}, \ell_{2}, \ell_{3}\right]$. Then $\left[\ell_{1}, \ell_{2}\right.$, $\left.\ell_{3}\right]$ is not of the form $[0,0, k]$ or $[0,1, k]$ for $k \geq 0$.

Proof. We begin by noticing that there can be no two consecutive zeros because otherwise the patch would be linear and could not contain three pentagons. Now, assume that $\Pi$ is a patch with side parameters $[0,1, k]$ for some $k>0$. The face on the side of length 0 must be a hexagon (because otherwise the patch would be linear), so we delete that face to create a new patch with side parameters $[1,0, k-1]$. We continue deleting the the face on the side of length 0 until we reach a patch with side parameters $[0,1,0]$ which is an impossible configuration to realize.

Lemma 2.5. If $\Pi$ is a pseudoconvex patch with side parameters $\left[\ell_{1}, \ell_{2}\right]$, then $\ell_{1}+\ell_{2} \geq 6$ or $\left[\ell_{1}, \ell_{2}\right] \in\{[2,2],[1,4],[2,3]\}$. If $\Pi$ is a pseduoconvex patch with side parameter $\left[\ell_{1}\right]$, then $\ell_{1} \geq 5$.

Proof. In the case where $\Pi$ has four pentagons, we know from [3] that the minimal pseudoconvex patches have side parameters $[2,2]$ and $[2,3]$. Thus, we only need to check that the patches with parameters $[1,3],[0,4]$, and $[0,5]$ are not realizable. Consider a patch with side parameters $[1,3]$. If one of the corner faces is a pentagon, we remove it to get a patch with three pentagons and parameters $[1,0,2]$ which is not possible. If neither corner is a pentagon, then the side of length 1 is only hexagons and we strip off this side yielding a $[2,1]$ patch which is not realizable.

A patch with side parameters $[0,4]$ (similarly $[0,5]$ ) must have a hexagon on the side of length 0 . Removing this face results in a patch with side parameters $[1,2]$ (or $[1,3]$ ) which is not possible. If $\Pi$ has 5 pentagons, we also refer to [3] to see that the minimal such patch has side length 5 . 

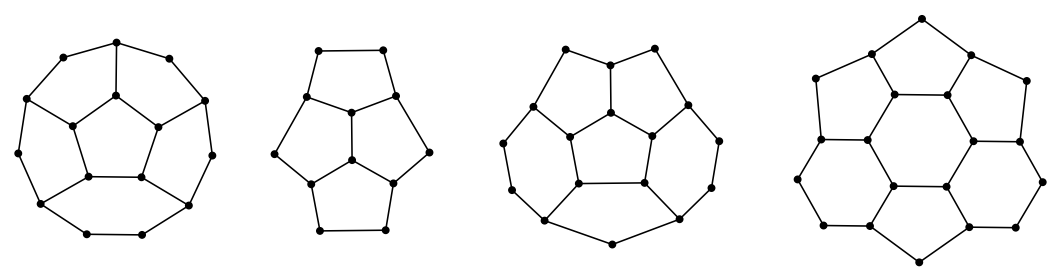

Figure 2: Patches with boundary codes $[5],[2,2],[2,3]$, and $[2,4]$ respectively.

\section{Sufficient conditions on side parameters}

The goal of this section is prove that all necessary conditions stated in the previous section are actually sufficient conditions. This direction is much more difficult because we will be working with a sequence of numbers that satisfy some conditions which we must then use to construct a patch. We begin constructing a patch that has five pentagons.

Lemma 3.1. There exists a pseudoconvex patch with side parameters $\left[\ell_{1}\right]$ for all $\ell_{1} \geq 5$.

Proof. If $\ell_{1}=5$, then the desired patch can be seen in Figure 2. For $\ell_{1}=5+k$ with $k \geq 1$, we add $k$ rings of hexagons to the patch in Figure 2 to yield a patch with side parameters $\left[\ell_{1}\right]$.

The next step is to construct pseudoconvex patches with four pentagons. Given a patch with side parameters $\left[\ell_{1}, \ell_{2}\right]$ with $\ell_{1}>0$, we see that adding a row of hexagons to the $\ell_{1}$ side creates a new patch with side parameters $\left[\ell_{1}-1, \ell_{2}+2\right]$. This technique will be used in the next proof.

Lemma 3.2. There exists a pseudoconvex patch with side parameters $\left[\ell_{1}, \ell_{2}\right]$ when $\ell_{1}+$ $\ell_{2} \geq 6$ or when $\left[\ell_{1}, \ell_{2}\right] \in\{[2,2],[1,4],[2,3]\}$.

Proof. Three small cases $([2,2],[2,3]$, and $[2,4])$ are shown in Figure 2. To draw the rest, we first assume that $\ell_{1} \leq \ell_{2}$. Let $d=\left(\ell_{2}-\ell_{1}\right) \bmod 3$. Write $\ell_{2}-\ell_{1}=3 k+d$. Starting with $[2,2+d]$, add a row of hexagons to the side of length 2 followed by a row of hexagons to the side of length $2+d$. Continue adding these two rows a total of of $\ell_{1}+k-2$ times. This yields the patch $\left[\ell_{1}+k, \ell_{1}+k+d\right]$. Now add $k$ rows of hexagons to the $\ell_{1}+k+d$ side to get a patch with side parameters $\left[\ell_{1}, \ell_{1}+3 k+d\right]=\left[\ell_{1}, \ell_{2}\right]$.

Before creating patches with three pentagons based on their side parameters, it is useful to remember how adding rows of hexagons changes the side parameters of a patch with three pentagons. Consider a patch with side parameters $\left[\ell_{1}, \ell_{2}, \ell_{3}\right]$. Notice that if we want to add a row of hexagons to the $\ell_{1}$ side of this patch, we need $\ell_{1}$ to be greater than 0 , and the new patch has side parameters $\left[\ell_{1}-1, \ell_{2}+1, \ell_{3}+1\right]$. If we want to add a row of hexagons to two sides at once, this is also possible. In this case, we already know from Lemma 2.4 that no two of the sides can be 0 . If only one of the sides is 0 , we can still add to both sides at the same time by essentially adding to the nonzero side first. Thus, adding a row of hexagons to both the $\ell_{1}$ side and the $\ell_{2}$ side of a patch with parameters $\left[\ell_{1}, \ell_{2}, \ell_{3}\right]$ results in a new patch with parameters $\left[\ell_{1}, \ell_{2}, \ell_{3}+2\right]$. This last operation will be especially important because it allows us to keep two sides of a patch the same size while increasing the length of the third side. 

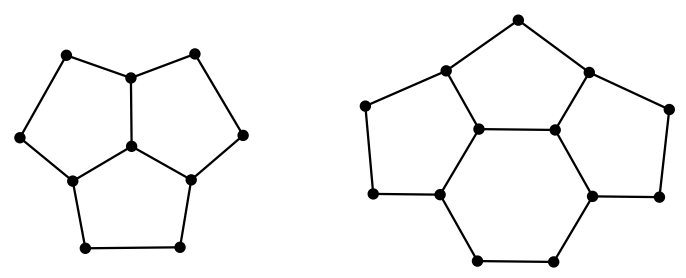

Figure 3: Patches with three pentagons and boundary codes $[1,1,1]$ and $[1,1,2]$.

Lemma 3.3. There exists a pseudoconvex patch with side parameters $\left[\ell_{1}, \ell_{2}, \ell_{3}\right]$ if $\left[\ell_{1}, \ell_{2}\right.$, $\left.\ell_{3}\right]$ is not of the form $[0,0, k]$ or $[0,1, k]$ for $k \geq 0$.

Proof. We begin by showing the patches with parameters $[1,1,1]$ and $[1,1,2]$ which are depicted in Figure 3. From these two figures we can make a few other essential patches. A $[2,2,1]$ patch can be constructed by adding a row of hexagons to a side of length 1 in the $[1,1,2]$ patch. A patch with side parameters $[2,2,2]$ can be constructed from the $[1,1,1]$ patch by adding rows of hexagons to all three sides. A $[0,2,2]$ patch can be constructed by adding a row of hexagons on one side of the $[1,1,1]$ patch. A patch with side parameters $[0,2,3]$ can be constructed by adding a row of hexagons to a side of length 1 in the $[1,1,2]$ patch. A $[0,3,3]$ patch can be constructed by adding two rows of hexagons to the side of length 2 on the $[1,1,2]$ patch. For the general construction, assume $\left[\ell_{1}, \ell_{2}, \ell_{3}\right]$ is not of the form $[0,0, k]$ or $[0,1, k]$ for $k \geq 0$ and that $\ell_{1}$ is the smallest side parameter.

If $\ell_{1}$ is nonzero, consider the patch $\left[S_{1}, S_{2}, S_{3}\right]$ where $S_{i}=\left(1+\ell_{i}\right) \bmod 2+1$ that was constructed above. For $i=1,2$, and 3 add $\left\lfloor\frac{\ell_{i}-1}{2}\right\rfloor$ rows of hexagons to the two sides $S_{j}$ where $j \neq i$ thus keeping the lengths of two sides fixed while increasing the length of the $S_{i}$ side by $2\left\lfloor\frac{\ell_{i}-1}{2}\right\rfloor$. Then the $i$ th side of the new patch has length

$$
\left(1+\ell_{i}\right) \bmod 2+1+2\left\lfloor\frac{\ell_{i}-1}{2}\right\rfloor=\ell_{i} .
$$

If $\ell_{1}=0$, consider the patch $\left[0, S_{2}, S_{3}\right]$ where $S_{i}=\ell_{2} \bmod 2+2$ that was constructed in the beginning of proof. For $i=2$ and $i=3$, add $\left\lfloor\frac{\ell_{i}-2}{2}\right\rfloor$ rows of hexagons to the two sides $S_{j}$ where $j \neq i$ (making sure to add to the nonzero side first). Adding these rows to two sides keeps the length of those two sides fixed while the $i$ th side of the new patch has length

$$
\ell_{i} \bmod 2+2+2\left\lfloor\frac{\ell_{i}-2}{2}\right\rfloor=\ell_{i} .
$$

It still remains to be shown that the necessary conditions on the patches with one or two pentagons are also sufficient conditions. Before creating patches with two pentagons, we examine how adding rows of hexagons changes the side parameters of such a patch. Consider a patch with side parameters $\left[\ell_{1}, \ell_{2}, \ell_{3}, \ell_{4}\right]$. Notice that the conditions in Lemma 2.3 imply that there are not two consecutive 0 's. If we want to add one row of hexagons to three sides of this patch at once, we can think of this as adding a row to a nonzero side first and then the other two sides. If the three sides we add a row to are the $\ell_{1}, \ell_{2}$, and $\ell_{3}$ side, then our new patch has side parameters $\left[\ell_{1}, \ell_{2}+1, \ell_{3}, \ell_{4}+2\right]$. 
Lemma 3.4. Let $\left[\ell_{1}, \ell_{2}, \ell_{3}, \ell_{4}\right]$ be a sequence of numbers satisfying $2 \ell_{i}+\ell_{i+1}+\ell_{i+3}>\ell_{i+2}$ where $i$ is cyclic in 1 to 4 . Then there exists a pseudoconvex patch with side parameters $\left[\ell_{1}, \ell_{2}, \ell_{3}, \ell_{4}\right]$.

Proof. Because the side parameters are cyclic and invertible, we can rearrange the side parameters so that $\ell_{1} \geq \ell_{3}, \ell_{2} \geq \ell_{4}$, and $2 \ell_{3}-\ell_{1} \leq 2 \ell_{4}-\ell_{2}$. We say the parameters are of Type $k$ if $\ell_{1}+\ell_{3}-\ell_{2}-\ell_{4}=k \bmod 3$. We also introduce the notation

$$
r_{i}=\frac{2 \ell_{i-2}+\ell_{i-1}+\ell_{i+1}-\ell_{i}}{3}
$$

for $i$ from 1 to 4 where indices are cyclic in 1 through 4 . The hypotheses of the lemma tell us that all $r_{i}$ 's are positive and are only integers for Type 0 conditions. Also, $\ell_{2} \geq \ell_{4}$ implies that $r_{2} \leq r_{4} ; \ell_{1} \geq \ell_{3}$ implies that $r_{1} \leq r_{3}$; and $2 \ell_{3}-\ell_{1} \leq 2 \ell_{4}-\ell_{2}$ implies that $r_{3} \geq r_{4}$. There are two essential patches that we will build our new patch from. These patches have side parameters $[1,1,1,1]$ and $[1,0,1,0]$ and are shown in Figure 4 . The overall construction will be to add either $\left\lfloor r_{i}-1\right\rfloor$ or $\left\lfloor r_{i}\right\rfloor$ rows of hexagons to a $j$ th side of an essential patch depending on these conditions. We must take some care to make sure we are never adding rows of hexagons to a side of length 0 .

Start by drawing the patch $[1,1,1,1]$ for Type 0 conditions, $[0,1,0,1]$ for Type 1 conditions, and $[1,0,1,0]$ for Type 2 conditions. Label the sides of the patch $S_{1}, S_{2}, S_{3}$, and $S_{4}$ in cyclic order.

We begin modifying these essential patches by first adding rows of hexagons to sides $S_{2}, S_{3}$, and $S_{4}$. Under Type 0 conditions we add $\left\lfloor r_{2}-1\right\rfloor$ rows whereas we add $\left\lfloor r_{2}\right\rfloor$ for Type 1 or 2 conditions. This new patch has side parameters

$$
\begin{gathered}
{\left[2 r_{2}-1,1, r_{2}, 1\right] \text { for Type } 0 \text { conditions, }} \\
{\left[2 r_{2}-\frac{2}{3}, 1, r_{2}-\frac{1}{3}, 1\right] \text { for Type } 1 \text { conditions, }} \\
{\left[2 r_{2}-\frac{1}{3}, 0, r_{2}+\frac{1}{3}, 0\right] \text { for Type } 2 \text { conditions. }}
\end{gathered}
$$

Next we add $r_{4}-r_{2}$ rows of hexagons to the $S_{3}$ and $S_{4}$ sides of the patch creating a patch with side parameters

$$
\begin{gathered}
{\left[r_{4}+r_{2}-1, r_{4}-r_{2}+1, r_{2}, 1\right] \text { for Type } 0 \text { conditions, }} \\
{\left[r_{4}+r_{2}-\frac{2}{3}, r_{4}-r_{2}+1, r_{2}-\frac{1}{3}, 1\right] \text { for Type } 1 \text { conditions, }} \\
{\left[r_{4}+r_{2}-\frac{1}{3}, r_{4}-r_{2}, r_{2}+\frac{1}{3}, 0\right] \text { for Type } 2 \text { conditions. }}
\end{gathered}
$$

The next step is to add $\left\lfloor r_{3}\right\rfloor-\left\lfloor r_{4}\right\rfloor$ rows of hexagons to the $S_{3}$ side of the patch yielding a patch with side parameters

$$
\begin{aligned}
& {\left[r_{4}+r_{2}-1, r_{3}-r_{2}+1, r_{2}-r_{3}+r_{4}, r_{3}-r_{4}+1\right] \text { for Type } 0 \text { conditions, }} \\
& {\left[r_{4}+r_{2}-\frac{2}{3}, r_{3}-r_{2}+\frac{2}{3}, r_{2}-r_{3}+r_{4}, r_{3}-r_{4}+\frac{2}{3}\right] \text { for Type } 1 \text { conditions, }} \\
& {\left[r_{4}+r_{2}-\frac{1}{3}, r_{3}-r_{2}+\frac{1}{3}, r_{2}-r_{3}+r_{4}, r_{3}-r_{4}+\frac{1}{3}\right] \text { for Type } 2 \text { conditions. }}
\end{aligned}
$$



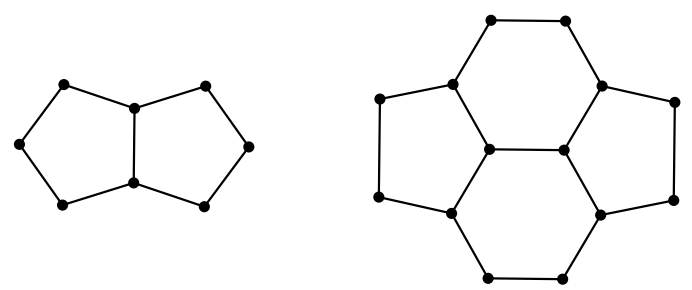

Figure 4: Patches with two pentagons and boundary sequences $[1,0,1,0]$ and $[1,1,1,1]$.

Note that the inequalities on $r_{i}$ 's allow us to decrease the third side parameter as needed.

Finally, we add rows of hexagons to $S_{1}$. We add $\left\lfloor r_{1}-1\right\rfloor$ rows under Type 0 conditions and $\left\lfloor r_{1}\right\rfloor$ rows under Type 1 or 2 conditions. Note that the inequalities on $r_{i}$ 's allow us to decrease the first side parameter as needed. Under any conditions the ith side becomes $r_{i-1}+r_{i+1}-r_{i}=\ell_{i}$, thus our new patch has side parameters $\left[\ell_{1}, \ell_{2}, \ell_{3}, \ell_{4}\right]$ as desired.

We now consider pseudoconvex patches with one pentagon. This construction of such patches will be similar to the previous proof. Consider a patch that has $\left[\ell_{1}, \ell_{2}, \ell_{3}, \ell_{4}, \ell_{5}\right]$ as its side parameters with either $\ell_{1}$ or $\ell_{2}$ being nonzero. Then we can add a row of hexagons to these two adjacent sides by adding to the nonzero side first creating a new patch with side parameters $\left[\ell_{1}, \ell_{2}, \ell_{3}+1, \ell_{4}, \ell_{5}+1\right]$. If both $\ell_{1}$ and $\ell_{2}$ are zero, then we have a linear patch with parameters $\left[0,0, \ell_{3}, 0, \ell_{3}\right]$. In order to add a row of hexagons to the $\ell_{1}$ and $\ell_{2}$ side, we simply add a hexagon to the end of the linear patch creating a new patch with parameters $\left[0,0, \ell_{3}+1,0, \ell_{3}+1\right]$. This idea of adding rows of hexagons to two sides simultaneously plays a large role in the following proof.

Lemma 3.5. Let $\ell_{1}, \ell_{2}, \ell_{3}, \ell_{4}$, and $\ell_{5}$ be given nonnegative integers satisfying the conditions that $\ell_{i}+\ell_{i+1}-\ell_{i+3} \geq 0$ for $i$ from 1 to 5 where subscripts are cyclic in 1 to 5 . Then there is a pseudoconvex patch with side parameters $\left[\ell_{1}, \ell_{2}, \ell_{3}, \ell_{4}, \ell_{5}\right]$.

Proof. Without loss of generality, we assume that $\ell_{1}$ is the largest of the side parameters and that $\ell_{2} \geq \ell_{5}$. We begin by drawing a single pentagon which is a patch with side parameters $[0,0,0,0,0]$. Label the sides of this patch $S_{1}, S_{2}, S_{3}, S_{4}$, and $S_{5}$ in cyclic order. The overall construction will be to add $\ell_{i+2}+\ell_{i+3}-\ell_{i}$ rows of hexagons to side $i$ of the single pentagon patch.

We begin by adding $\ell_{3}+\ell_{4}-\ell_{1}$ rows of hexagons to the $S_{1}$ and $S_{5}$ sides of the patch creating a patch with side parameters

$$
\left[0, \ell_{3}+\ell_{4}-\ell_{1}, 0, \ell_{3}+\ell_{4}-\ell_{1}, 0\right]
$$

We then add $\left(\ell_{2}-\ell_{5}\right)+\left(\ell_{1}-\ell_{4}\right)$ rows of hexagons to sides $S_{4}$ and $S_{5}$ yielding a patch with side parameters

$$
\left[\ell_{2}-\ell_{5}+\ell_{1}-\ell_{4}, \ell_{3}+\ell_{4}-\ell_{1}, \ell_{2}-\ell_{5}+\ell_{1}-\ell_{4}, \ell_{3}+\ell_{4}-\ell_{1}, 0\right] .
$$

The next step is to add $\ell_{5}$ rows of hexagons to the $S_{3}$ and $S_{4}$ sides which creates a patch with side parameters

$$
\left[\ell_{2}-\ell_{5}+\ell_{1}-\ell_{4}, \ell_{3}+\ell_{4}+\ell_{5}-\ell_{1}, \ell_{2}-\ell_{5}+\ell_{1}-\ell_{4}, \ell_{3}+\ell_{4}-\ell_{1}, \ell_{5}\right] .
$$


There are now two cases to consider. The first case is if $\ell_{1}+\ell_{2} \leq \ell_{3}+\ell_{4}+\ell_{5}$. In this case we add $\ell_{1}-\ell_{3}$ rows of hexagons to sides $S_{2}$ and $S_{3}$. This patch has side parameters

$$
\left[2 \ell_{1}+\ell_{2}-\ell_{3}-\ell_{4}-\ell_{5}, \ell_{3}+\ell_{4}+\ell_{5}-\ell_{1}, \ell_{1}+\ell_{2}-\ell_{4}-\ell_{5}, \ell_{4}, \ell_{5}\right]
$$

To this new patch, we add $\ell_{3}+\ell_{4}+\ell_{5}-\ell_{1}-\ell_{2}$ rows of hexagons to side $S_{2}$ creating a patch with side parameters

$$
\left[\ell_{1}, \ell_{2}, \ell_{3}, \ell_{4}, \ell_{5}\right]
$$

For the case where $\ell_{1}+\ell_{2}>\ell_{3}+\ell_{4}+\ell_{5}$, we start with the patch with side parameters

$$
\left[\ell_{2}-\ell_{5}+\ell_{1}-\ell_{4}, \ell_{3}+\ell_{4}+\ell_{5}-\ell_{1}, \ell_{2}-\ell_{5}+\ell_{1}-\ell_{4}, \ell_{3}+\ell_{4}-\ell_{1}, \ell_{5}\right]
$$

that we created above. At this point we add $\ell_{4}+\ell_{5}-\ell_{2}$ rows of hexagons to the $S_{2}$ and $S_{3}$ sides of the patch creating the patch with side parameters

$$
\left[\ell_{1}, \ell_{3}+\ell_{4}+\ell_{5}-\ell_{1}, \ell_{2}-\ell_{5}+\ell_{1}-\ell_{4}, \ell_{3}+2 \ell_{4}+\ell_{5}-\ell_{1}-\ell_{2}, \ell_{5}\right] .
$$

Finally, adding $\ell_{1}+\ell_{2}-\ell_{3}-\ell_{4}-\ell_{5}$ rows of hexagons to the $S_{3}$ side creates the desired patch.

All of our results are summarized in the following theorem.

Theorem 3.6. The sequence of nonnegative integers $\left[\ell_{1}, \ldots, \ell_{s}\right]$ are the side parameters of a pseudoconvex patch consisting of $6-s$ pentagons if and only if one of the following is true:

1. $s=1$ and $\ell_{1} \geq 5$.

2. $s=2$ and $\left[\ell_{1}, \ell_{2}\right] \in\{[2,2],[1,4],[2,3]\}$ or $\ell_{1}+\ell_{2} \geq 6$

3. $s=3$ and the side parameters are not of the form $[0,1, k]$ or $[0,0, k]$ for $k \geq 0$.

4. $s=4$ and there are no two consecutive zeros and the following four inequalities are satisfied:

(a) $2 \ell_{1}+\ell_{2}+\ell_{4}>\ell_{3}$

(b) $2 \ell_{2}+\ell_{3}+\ell_{1}>\ell_{4}$

(c) $2 \ell_{3}+\ell_{4}+\ell_{2}>\ell_{1}$

(d) $2 \ell_{4}+\ell_{1}+\ell_{3}>\ell_{2}$

5. $s=5$ and the following five inequalities are satisfied:

(a) $\ell_{1}+\ell_{2} \geq \ell_{4}$

(b) $\ell_{2}+\ell_{3} \geq \ell_{5}$

(c) $\ell_{3}+\ell_{4} \geq \ell_{1}$

(d) $\ell_{4}+\ell_{5} \geq \ell_{2}$

(e) $\ell_{5}+\ell_{1} \geq \ell_{3}$

6. $s=6$ and the following three equalities are satisfied: 
(a) $\ell_{1}+\ell_{2}=\ell_{4}+\ell_{5}$

(b) $\ell_{2}+\ell_{3}=\ell_{5}+\ell_{6}$

(c) $\ell_{3}+\ell_{4}=\ell_{6}+\ell_{1}$

As a corollary, we state this theorem in terms of boundary codes.

Corollary 3.7. The boundary code $2(23)^{\ell_{1}} 2(23)^{\ell_{2}} \cdots 2(23)^{\ell_{s}}$ is the boundary code for a fullerene patch if and only if one of the conditions (1)-(6) in the previous theorem is satisfied.

\section{References}

[1] P. Bonsma and F. Breuer, Finding fullerene patches in polynomial time, in ISAAC 2009, volume 5878 of $L N C S$ (2009), 750-759.

[2] J. Bornhoft, G. Brinkmann and J. Greinus, Pentagon-hexagon-patches with short boundaries, Eur. J. Combin. 24 (2003), 517-529.

[3] G. Brinkmann and N. Van Cleemput, Classification and Generation of Nanocones, Discrete Appl. Math. 159 (2011), 1528-1539.

[4] M. Deza, P. W. Fowler and V. Grishukhin, Allowed boundary sequences for fused polycyclic patches and related algorithmic problems, J. Chem. Inf. Comput. Sci. 41 (2001), 300-308.

[5] J. E. Graver, The $(m, k)$-patch boundary code problem, MATCH Commun. Math. Comput. Chem. 48 (2003), 189-196.

[6] J. E. Graver and G. Cargo, When does a curve bound a distorted disk?, SIAM J. Discrete Math. 25 (2011), 280-305.

[7] J. E. Graver and C. Graves, Fullerene Patches I, Ars Math. Contemp. 3 (2010), 109-120.

[8] X. Guo, P. Hansen and M. Zheng, Boundary Uniqueness of Fusenes, Discrete Appl. Math. 118 (2002), 209-222.

[9] D. J. Klein and A. T. Balaban, The Eight Classes of Positive-Curvature Graphic Nanocones, J. Chem. Inf. Model. 46 (2006), 307-320. 